\title{
Neuroimaging-based brain-age prediction in diverse forms of epilepsy: a signature of psychosis and beyond
}

\author{
Daichi Sone ${ }^{1,2} \cdot$ Iman Beheshti $^{1} \cdot$ Norihide Maikusa $^{1} \cdot$ Miho Ota $^{1,3} \cdot$ Yukio Kimura ${ }^{4} \cdot$ Noriko Sato $^{4}$. \\ Matthias Koepp ${ }^{2} \cdot$ Hiroshi Matsuda ${ }^{1}$
}

Received: 10 January 2019 / Revised: 17 April 2019 / Accepted: 3 May 2019 / Published online: 3 June 2019

(c) The Author(s) 2019. This article is published with open access

\begin{abstract}
Epilepsy is a diverse brain disorder, and the pathophysiology of its various forms and comorbidities is largely unknown. A recent machine learning method enables us to estimate an individual's "brain-age" from MRI; this brain-age prediction is expected as a novel individual biomarker of neuropsychiatric disorders. The aims of this study were to estimate the brain-age for various categories of epilepsy and to evaluate clinical discrimination by brain-age for (1) the effect of psychosis on temporal lobe epilepsy (TLE), (2) psychogenic nonepileptic seizures (PNESs) from MRI-negative epilepsies, and (3) progressive myoclonic epilepsy (PME) from juvenile myoclonic epilepsy (JME). In total, 1196 T1-weighted MRI scans from healthy controls (HCs) were used to build a brain-age prediction model with support vector regression. Using the model, we calculated the brain-predicted age difference (brain-PAD: predicted age-chronological age) of the HCs and 318 patients with epilepsy. We compared the brain-PAD values based on the research questions. As a result, all categories of patients except for extra-temporal lobe focal epilepsy showed a significant increase in brain-PAD. TLE with hippocampal sclerosis presented a significantly higher brain-PAD than several other categories. The mean brain-PAD in TLE with interictal psychosis was 10.9 years, which was significantly higher than TLE without psychosis (5.3 years). PNES showed a comparable mean brain-PAD (10.6 years) to that of epilepsy patients. PME had a higher brain-PAD than JME (22.0 vs. 9.3 years). In conclusion, neuroimaging-based brain-age prediction can provide novel insight into or clinical usefulness for the diverse symptoms of epilepsy.
\end{abstract}

These authors contributed equally: Daichi Sone, Iman Beheshti, Norihide Maikusa

Supplementary information The online version of this article (https:// doi.org/10.1038/s41380-019-0446-9) contains supplementary material, which is available to authorized users.

Daichi Sone

d.sone@ucl.ac.uk

1 Integrative Brain Imaging Center, National Center of Neurology and Psychiatry, Tokyo, Japan

2 Department of Clinical and Experimental Epilepsy, UCL Institute of Neurology, London, UK

3 Department of Neuropsychiatry, Division of Clinical Medicine, Faculty of Medicine, University of Tsukuba, Ibaraki, Japan

4 Department of Radiology, National Center of Neurology and Psychiatry, Tokyo, Japan

\section{Introduction}

Epilepsy is a common but quite diverse brain disorder [1]. Attempts at clinical classification of the disease are continuing, considering the many types of seizures, electroencephalogram (EEG) findings, structural abnormalities, and genetics [2]. In addition, although epileptic seizure is the main symptom of epilepsy, various forms of comorbidities often eventually occur in patients with epilepsy [3]. In particular, psychiatric and behavioral problems, including psychogenic nonepileptic seizures (PNESs), are one of the significant aspects of the condition [4]. However, the pathophysiology of these diverse forms of epilepsy and its related disorders is still unclear and needs to be elucidated for better clinical treatments.

On the other hand, machine learning in brain images has recently become expected to be used as a potential individual-level biomarker in many neuropsychiatric disorders [5]. There are already useful applications for automatic categorization of epilepsy neuroimaging [6]. In 
addition, more recent advances in machine learning have allowed us to predict the age of an individual's brain image using regression models [7]. This "neuroimaging-based brain-age prediction" has been applied to several psychiatric disorders and Alzheimer's disease [7, 8]. As for epilepsy, Pardoe et al. reported a significant 4.5-year increase in brain age compared with chronological age in refractory focal epilepsy [9]. We considered that the brain-age prediction could provide useful information on other forms of epilepsy. Thus, the initial aim of this study was to estimate brain age in various categories of epilepsy in accordance with the following three clinical research questions.

First is the effect of psychosis in epilepsy. Psychosis, which is an important comorbidity of epilepsy, is found in around $10-20 \%$ of patients with temporal lobe epilepsy (TLE), which is higher than in the general population [10]. On the other hand, many other TLE patients do not develop psychosis, and no plausible explanation has yet been proposed for these different phenotypes. According to recent studies of brain age in schizophrenia $[8,11]$, patients with schizophrenia show a 3- to 5-year higher brain age compared with chronological age, and this gap accelerates around the onset of psychosis. Thus, we hypothesized that brain age could also be a novel biomarker in psychosis of epilepsy and possibly show higher values than in nonpsychotic patients.

Second, we aimed to investigate the differences between PNES and epilepsy with no lesion on visual assessment of MRI (i.e., magnetic resonance imaging (MRI)-negative epilepsy). PNES is defined as episodes resembling epileptic seizures but caused by psychogenic mechanisms [12]. Because PNES does not have epileptic physiology, patients with PNES should be treated differently from patients with epilepsy. Nevertheless, the diagnosis of PNES is sometimes difficult, especially with limited access to video-EEG monitoring as the goldstandard diagnosis of PNES confirming ictal semiology and EEG findings [12]. Actually, PNES is recognized as a diagnostic and therapeutic problem in many countries [13]. We considered that an MRI-based biomarker that could identify significant differences between PNES and epilepsy, particularly in visually normal MRI cases, would be helpful for clinicians and patients. Moreover, the results may shed additional light on the pathophysiology of PNES, although PNES is heterogeneous and cannot be explained by any single mechanism [14].

Third, we addressed the differences between progressive myoclonic epilepsy (PME) and juvenile myoclonic epilepsy (JME). PME is a group of neurodegenerative disorders characterized by myoclonic seizures and progressive neurological impairment, including ataxia or intellectual deterioration [15]. Because PME is often difficult to clinically distinguish from JME at the early stage, which is a more common and basically nonprogressive group with much better prognosis, some attempts have been made to demonstrate specific differences between PME and JME [16]. As with the second aim, we also applied brain-age prediction to this differentiation.

\section{Materials and Methods}

\section{Healthy controls}

To build and estimate the brain-age model, we recruited 1196 MRI scans at our center from healthy controls (HCs) with no history of neurological or psychiatric diseases and no use of medication affecting the central nervous system. No possible structural anomalies or abnormalities affecting the analysis were visually found in the controls on MRI. The 1196 HCs were aged between 20 and 89 years (mean \pm SD: $55.4 \pm 15.3$ years) and comprised 426 men and 770 women. The mean ages and proportions of sex were different between each group of patients, but the HC database contained sufficient samples of each age and sex. Because epilepsy can affect individuals of all ages, we decided to include all available samples to establish a reliable brainage model.

\section{Patients}

Of the 437 adult (i.e., $\geq 20$-year-old) patients with epilepsy or PNES recruited at our institute between November 2013 and December 2017, we enrolled 318 patients in the brain age analyses based on the following examinations and criteria.

All of the patients underwent careful clinical diagnosis by board-certified clinical epileptologists based on seizure semiology and conventional scalp EEG and conventional 3.0-T MRI inspection by experienced neuroradiologists. More detailed inclusion criteria and specific examinations performed for each category of epilepsy are described in Table 1.

The initial categorization of epilepsy at this stage was as follows: (1) TLE with no visible lesion (i.e., visually normal) on MRI (TLE-NL), (2) TLE with hippocampal sclerosis (TLE-HS), (3) extra-temporal lobe focal epilepsy (Ext-FE), (4) idiopathic generalized epilepsy (IGE), (5) PME or symptomatic generalized epilepsy (PME/SGE), and (6) PNES without any epileptic seizures (PNES).

The secondary categorization included psychosis vs. nonpsychosis in TLE, PNES vs. MRI-negative epilepsies, and JME vs. PME. The composition of MRI-negative epilepsies is shown in Supplementary Table 1. JME was diagnosed based on the presence of myoclonic seizures in addition to the criteria for IGE listed in Table 1. 


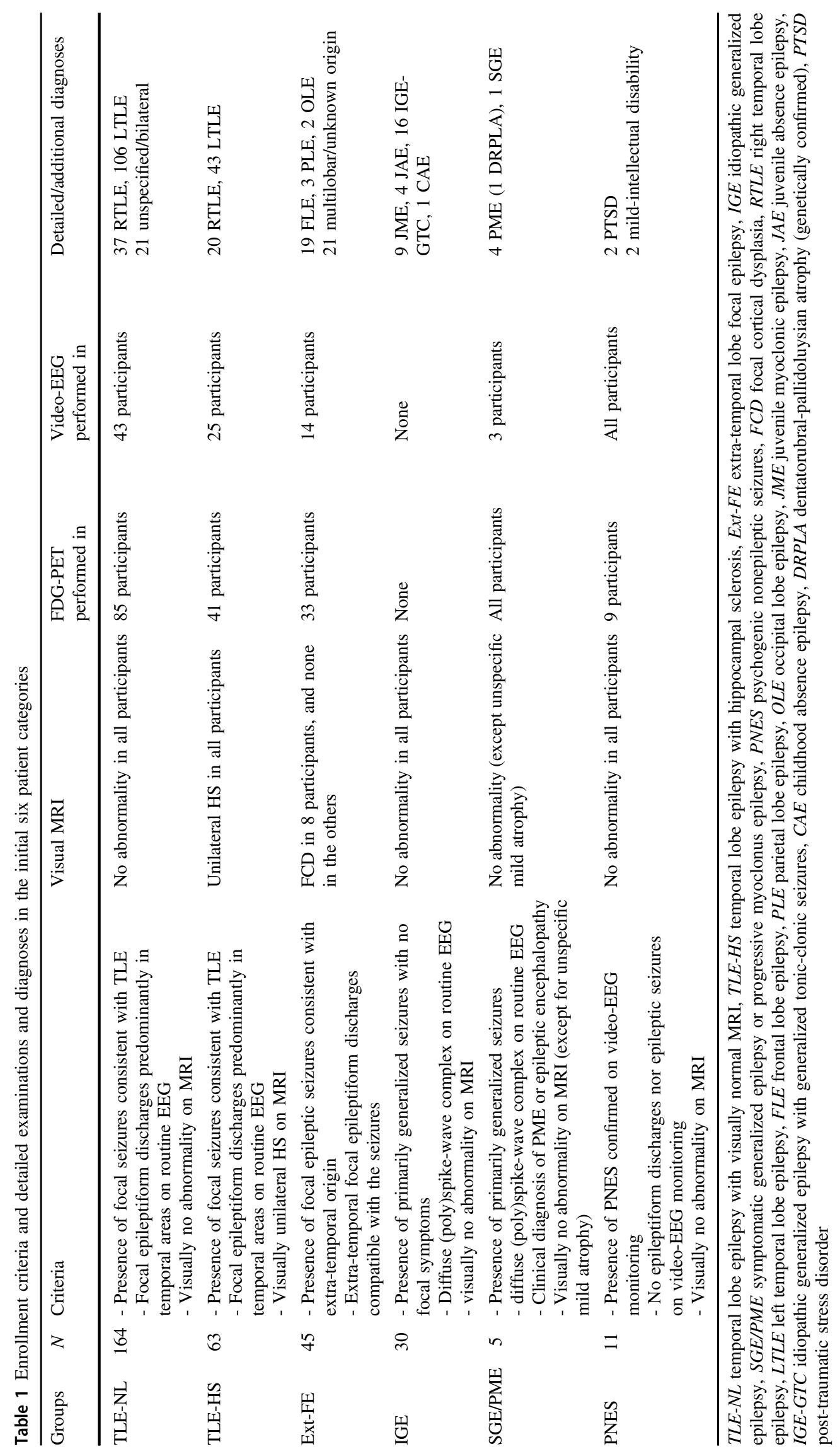


The following exclusion criteria were applied to all patients: (1) a significant medical history of acute encephalitis, meningitis, severe head trauma, ischemic encephalopathy, or brain surgery; and (2) suspicious epileptogenic lesions (e.g., tumor, vascular malformation, and destructive lesion) on MRI other than unilateral HS or focal cortical dysplasia (FCD).

In total, 119 of the initial 437 patients were not enrolled, including 43 with focal epileptogenic lesions other than HS or FCD, 37 who were not classified into any specific categories, 11 with a history of encephalitis, 8 with severe head trauma, and 20 with other reasons (e.g., postneurosurgery and poor imaging quality).

All participants gave written informed consent. The study was approved by the Institutional Review Board at the National Center of Neurology and Psychiatry Hospital.

\section{Psychosis evaluation}

We assessed the existence of inter-ictal psychosis (IIP) only in patients with TLE. Because TLE has the highest prevalence of psychosis, this investigation of psychosis was originally planned for TLE patients. The presence or history of IIP was diagnosed based on the Diagnostic and Statistical Manual of Mental Disorders, 4th edition criteria [17]. Of the 227 patients with TLE, 21 were diagnosed with IIP; the others had no psychotic episodes.

\section{MRI acquisition}

The three-dimensional (3D) sagittal T1-weighted images of the HCs were obtained from two different protocols on 3.0T MRI scanners: 798 individuals underwent Protocol 1 and the other 398 individuals underwent Protocol 2. On the other hand, all of the patients underwent Protocol 1.

Protocol 1: 3.0-T MR system (Philips Medical Systems, Best, The Netherlands) with the following protocol: repetition time (TR)/echo time (TE), $7.18 \mathrm{~ms} / 3.46 \mathrm{~ms}$; flip angle, $10^{\circ}$; number of excitations (NEX), $1 ; 0.68 \times 0.68 \mathrm{~mm}^{2}$ in plane resolution; 0.6-mm effective slice thickness with no gap; 300 slices; matrix, $384 \times 384$; field of view (FOV), $26.1 \times 26.1 \mathrm{~cm}$.

Protocol 2: 3.0-T MR system (Verio, Siemens, Erlangen, Germany) with the following protocol: TR/TE, $1800 \mathrm{~ms} /$ $2.25 \mathrm{~ms}$; flip angle, $9^{\circ}$; NEX, $1 ; 0.87 \times 0.78 \mathrm{~mm}^{2}$ in plane resolution; $0.8-\mathrm{mm}$ effective slice thickness with no gap; 224 slices; matrix, $320 \times 280$; FOV, $25 \times 25 \mathrm{~cm}$.

\section{Neuroimaging processing}

The pipeline of processing is described in Supplementary Fig. 1. Using SPM12 (Wellcome Trust Centre for Neuroimaging, London, UK; www.fl.ion.ucl.ac.uk/spm/), all 3D
T1-weighted images were bias-corrected and segmented into gray matter (GM), white matter (WM), and cerebrospinal fluid components. The GM and WM components were used. To ensure the accuracy of the image segmentation, all segmented GM and WM images were visually inspected. We then used SPM DARTEL [18] to conduct a nonlinear registration to a custom template on the basis of a training dataset (i.e., healthy individuals, $[N=1196])$. The imaged GM and WM were then registered to MNI space, modulated to retain tissue volume information, and smoothed with a 4-mm Gaussian kernel [19, 20]. As per the pipeline proposed in [21], the spatially normalized GM and WM images were resampled into $8 \mathrm{~mm}$ isotropic spatial resolution. For each individual, the voxel intensities extracted from smoothed GM and WM images were concatenated to build a whole-brain estimating age and considered as raw features for the regression model.

\section{Regression model and validation}

To explore the brain age in various forms of epilepsy, we used a standard nu-support vector regression (nu-SVR) model conducted in LIBSVM (http://www.csie.ntu.edu.tw/ $\sim$ cjlin/libsvm/) toolbox with linear kernel and default set of parameters (i.e., in the LIBSVM: $C=1, v=0.5$ ). SVR has previously shown a robust performance in estimating brain age from T1-weighted MRI images [22]. Following [21], a principal component analysis was used to reduce the probability of overfitting and overcome the curse of dimensionality. The number of principal components was set at 100 per individual.

Consequently, for the regression model, the chronological age was considered the dependent variable and the principal components derived from the concatenated GM and WM voxel intensities were considered the independent variables. To assess the ability of the proposed regression model, we conducted a tenfold cross validation on the training set (i.e., healthy individuals), with onefold in each iteration considered as the test and the remaining folds considered the training set. The model accuracy was measured via the mean absolute error, root mean squared error, and the correlation between the chronological age and estimated age through tenfold cross validation. Thereafter, the final regression model was built using the entire training set (i.e., healthy individuals $[N=1196])$ and then applied to epilepsy patients $(N=318)$ to estimate the brain ages.

\section{Group comparisons and correlations of the brain- predicted age difference}

Based on the age predicted by the MRI-based SVR model, we calculated each participant's brain-predicted age difference (brain-PAD: predicted age—chronological age). First, 
Table 2 Clinical demographics and brain-PAD results in the initial categorization of patients

\begin{tabular}{|c|c|c|c|c|c|c|}
\hline & $\begin{array}{l}\text { TLE-NL } \\
(N=164)\end{array}$ & $\begin{array}{l}\text { TLE-HS } \\
(N=63)\end{array}$ & $\begin{array}{l}\text { Ext-FE } \\
(N=45)\end{array}$ & $\begin{array}{l}\text { IGE } \\
(N=30)\end{array}$ & $\begin{array}{l}\text { SGE/PME } \\
(N=5)\end{array}$ & $\begin{array}{l}\text { PNES } \\
(N=11)\end{array}$ \\
\hline \multicolumn{7}{|l|}{ Age (y) } \\
\hline Mean \pm SD & $45.8 \pm 16.6$ & $43.3 \pm 13.7$ & $35.9 \pm 12.0$ & $28.9 \pm 7.7$ & $31.4 \pm 9.8$ & $31.5 \pm 8.6$ \\
\hline \multicolumn{7}{|l|}{$\operatorname{Sex}(N)$} \\
\hline Male:female & $81: 83$ & $25: 38$ & $27: 18$ & $8: 22$ & $3: 2$ & $3: 8$ \\
\hline \multicolumn{7}{|l|}{ Disease duration (y) } \\
\hline Mean onset age \pm SD & $30.8 \pm 20.8$ & $14.4 \pm 10.9$ & $13.3 \pm 10.5$ & $15.6 \pm 6.1$ & $10.6 \pm 8.7$ & $23.6 \pm 11.1$ \\
\hline Mean duration $\pm \mathrm{SD}$ & $15.0 \pm 13.9$ & $29.0 \pm 13.5$ & $22.6 \pm 12.6$ & $13.3 \pm 9.3$ & $20.8 \pm 15.7$ & $7.8 \pm 7.3$ \\
\hline \multicolumn{7}{|l|}{ Drug-resistance $(N, \%)$} \\
\hline $\begin{array}{l}\text { Patients with } \\
\text { refractory SZ }\end{array}$ & $149(91 \%)$ & $61(97 \%)$ & $43(96 \%)$ & $6(20 \%)$ & $5(100 \%)$ & $11(100 \%)$ \\
\hline \multicolumn{7}{|l|}{ Brain-age estimation (y) } \\
\hline Mean brain-PAD $\pm \mathrm{SD}$ & $4.7 \pm 7.9$ & $8.8 \pm 7.3$ & $5.6 \pm 7.5$ & $8.9 \pm 6.3$ & $21.2 \pm 10.0$ & $10.6 \pm 5.6$ \\
\hline $\begin{array}{l}\text { Estimated marginal } \\
\text { mean } \pm \mathrm{SE}\end{array}$ & $3.3 \pm 0.5$ & $6.9 \pm 0.8$ & $2.1 \pm 0.9$ & $3.8 \pm 1.1$ & $16.7 \pm 2.7$ & $6.1 \pm 1.8$ \\
\hline
\end{tabular}

$T L E-N L$ temporal lobe epilepsy with visually normal MRI, TLE-HS temporal lobe epilepsy with hippocampal sclerosis, Ext-FE extra-temporal lobe focal epilepsy, $I G E$ idiopathic generalized epilepsy, $S G E / P M E$ symptomatic generalized epilepsy or progressive myoclonic epilepsy, PNES psychogenic nonepileptic seizures, $S Z$ seizures, brain- $P A D$ brain-predicted age difference, $S E$ standard error we compared the mean brain-PAD among the six initial categories of patients and the HCs. In addition, correlations of the brain-PAD with disease duration or onset age were investigated in each initial category. For the three clinical differentiations, we evaluated the relevant two groups by comparing the mean brain-PAD and performing receiver operating characteristic (ROC) curve and area under the curve (AUC) analyses. However, we refrained from using inferential statistics for the comparison between JME and PME because of the small sample sizes of the two groups.

\section{Statistics}

Statistical analyses were performed by SPSS software, version 23.0 (SPSS Japan, Tokyo). The mean brain-PAD was compared via analysis of covariance (ANCOVA) with age and sex as covariates. Because onset age or disease duration in each group did not show a normal distribution, the correlations of the brain-PAD with these parameters were analyzed by a nonparametric method (i.e., Spearman's rank correlation coefficient) with Bonferroni correction for multiple comparisons. The ROC curves were nonparametrically analyzed according to whether the AUC was significantly higher than 0.5 (i.e., random) to differentiate the two relevant clinical categories. Other clinical parameters than brain-PAD were compared by an unpaired $t$ test for continuous variables and a Pearson's $\chi^{2}$ test for binary parameters. A $p$ value $<0.05$ was deemed significant.

This study included various types of analysis, and then not all analyses had the sample sizes validated. However, in the comparisons among initial categories, psychosis vs. nonpsychosis, and PNES vs. MRI-negative epilepsies, the total sample sizes were demonstrated to achieve $80 \%$ power to detect differences with 0.25 effect sizes based on G*Power 3.1.9.4 [23].

\section{Results}

\section{Clinical demographics}

The clinical demographics are presented in Table 2. Each group showed differing distributions of age, sex, and disease onset/duration. Most patients had refractory seizures except those in the IGE group.

\section{Brain-age prediction model in HCs}

Figure 1 contains each individual's predicted age and chronological age. The SVR brain-age prediction model showed a mean absolute error of 5.28 years in HCs, and the predicted age in $\mathrm{HCs}$ was highly correlated with their chronological age $(r=0.90)$. The mean $( \pm \mathrm{SD})$ brain-PAD in HCs was $0.13( \pm 6.7)$ years.

Because there are different age and sex distributions between the two MRI scanners (Supplementary Fig. 2a), we investigated the scanner effect for brain-PAD. Consequently, as in Supplementary Fig. 2b, the two scanners showed a similar pattern for brain-PAD among their HCs with no significant difference in the mean value $(p=0.299$, unpaired $t$ test). 


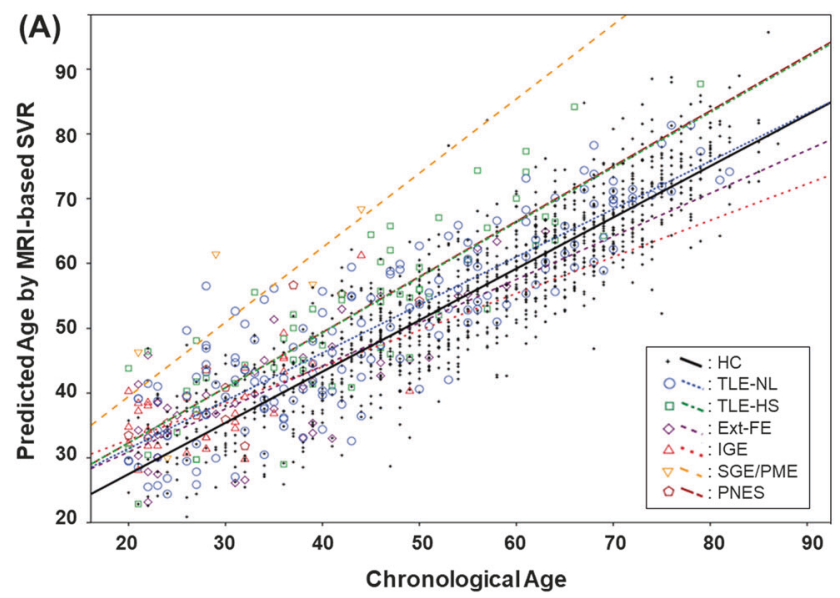

Fig. 1 a The scatter plots and linear fits of the individual predicted brain and chronological ages in each group. $\mathbf{b}$ The box plot of brainPAD in each group. $* p<0.05, * * p<0.01, * * * p<0.001,{ }^{\dagger} \mathrm{HCs}$ showed significantly lower brain-PAD than TLE-NL $(p<0.001)$, TLE-HS $(p<$

\section{Brain-PAD in the six categories of patients}

As per the initial aim of this study, we evaluated the brain-PAD of the initial categorizations of patients. All six categories of patients showed a higher mean brainPAD compared with HCs, and the differences were statistically significant for five of the groups, with the exception of Ext-FE $(p=0.149)$ (Table 2 and Fig. 1). The PME/SGE group had the highest brain-PAD and the TLE-HS group showed a significantly higher mean brainPAD than the TLE-NL group. The brain-PAD values corrected for age and sex (i.e., estimated marginal means by ANCOVA) are also shown in Table 2 and Supplementary Fig. 3a.

In addition, the onset age in TLE-NL was negatively correlated with the brain-PAD (Spearman's $r \mathrm{~s}=-0.436, p$ $<0.001$, Supplementary Fig. 3b). All other correlations were insignificant.

\section{Psychosis vs. nonpsychosis in TLE}

The clinical demographics and brain-PAD comparison results are shown in Table 3 and Fig. 2. The TLE with psychosis group showed a significantly higher brain-PAD than the TLE without psychosis group $(p<0.001$, ANCOVA). Because TLE with HS has higher brain-PAD, we added the existence of HS as covariate post hoc, but the significance remained $(p=0.005)$. There is no significant interaction for brain-PAD between the existence of HS and psychosis $(p=0.898)$. The detailed demographics of these categorizations are presented in Supplementary Table 2. The AUC was 0.694 for differentiating psychotic from nonpsychotic patients in TLE.
(B)

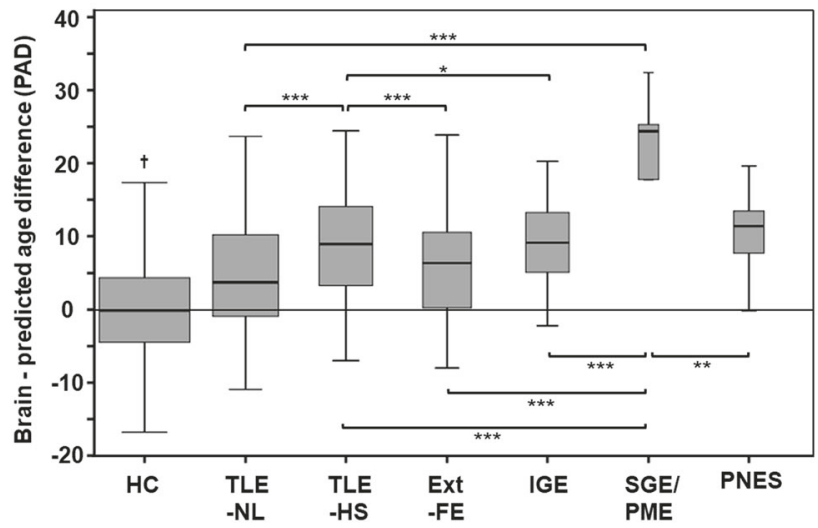

$0.001)$, IGE $(p<0.01)$, SGE/PME $(p<0.001)$, and PNES $(p<0.01)$, but the difference was not significant for Ext-FE $(p=0.134)$. The $p$ values are corrected by ANCOVA. The width of the boxes is weighted by the logarithm of the number of subjects in each group

\section{PNES vs. MRI-negative epilepsies}

This section compared 11 patients with PNES and 236 patients with MRI-negative epilepsies, including 164 TLENL, 37 MRI-negative cases from the Ext-FE group, 30 IGE, and 5 PME/SGE. The mean brain-PAD was not significantly different between PNES and MRI-negative epilepsies by ANCOVA comparison, although the PNES group had higher brain-PAD values (Table 3 and Fig. 2). On the other hand, the ROC curve of differentiation by the raw brain-PAD values showed an AUC of 0.700 .

\section{JME vs. PME}

We enrolled nine JME patients and four PME patients from the IGE and PME/SGE categories, respectively. The mean brain-PAD was higher in PME than in JME (Table 3 and Fig. 2).

\section{Discussion}

In this study, we performed neuroimaging-based brain-age prediction for various forms of epilepsy. The brain age in HCs was consistent across MRI scanners, which might suggest that the algorithm for brain age can provide a good biomarker beyond scanner differences. As the initial result, all categories of patients showed significantly increased brainage values, with only Ext-FE showing a statistical trend level value. In the six categories, SGE/PME, which is the most severe phenotype, showed the highest brain-PAD, and TLEHS, which is accompanied by distinct brain morphological changes, had higher values than other epilepsy syndromes. 
Table 3 Clinical demographics and brain-PAD results for each comparison

\begin{tabular}{|c|c|c|c|c|c|c|c|c|c|}
\hline & \multicolumn{3}{|c|}{ Psychosis vs. nonpsychosis in TLE } & \multicolumn{3}{|c|}{ PNES vs. MRI-negative epilepsies } & \multicolumn{3}{|c|}{ JME vs. PME } \\
\hline & $\begin{array}{l}\text { TLE-Non-P } \\
(N=206)\end{array}$ & $\begin{array}{l}\text { TLE-P } \\
(N=21)\end{array}$ & $p$ & $\begin{array}{l}\text { PNES } \\
(N=11)\end{array}$ & $\begin{array}{l}\operatorname{MRI}(-) \text { Epi } \\
(N=236)\end{array}$ & $p$ & $\begin{array}{l}\text { JME } \\
(N=9)\end{array}$ & $\begin{array}{l}\text { PME } \\
(N=4)\end{array}$ & $p$ \\
\hline \multicolumn{10}{|l|}{ Age (y) } \\
\hline Mean \pm SD & $45.1 \pm 16.3$ & $45.0 \pm 11.2$ & 0.976 & $31.5 \pm 8.6$ & $41.6 \pm 16.3$ & 0.003 & $24.1 \pm 4.6$ & $29.5 \pm 10.2$ & 0.202 \\
\hline \multicolumn{10}{|l|}{$\operatorname{Sex}(N)$} \\
\hline Male:female & $98: 108$ & $8: 13$ & 0.407 & $3: 8$ & $114: 122$ & 0.172 & $1: 8$ & $2: 2$ & 0.125 \\
\hline \multicolumn{10}{|l|}{ Disease duration (y) } \\
\hline Mean onset age \pm SD & $27.6 \pm 20.2$ & $12.3 \pm 9.8$ & $<0.001$ & $23.6 \pm 11.1$ & $25.9 \pm 19.5$ & 0.537 & $15.1 \pm 3.5$ & $11.3 \pm 9.9$ & 0.500 \\
\hline Mean duration $\pm \mathrm{SD}$ & $17.4 \pm 14.7$ & $32.7 \pm 11.5$ & $<0.001$ & $7.8 \pm 7.3$ & $15.7 \pm 13.2$ & 0.005 & $9.0 \pm 5.3$ & $18.3 \pm 16.8$ & 0.355 \\
\hline \multicolumn{10}{|l|}{ Drug-resistance $(N)$} \\
\hline $\begin{array}{l}\text { Patients with } \\
\text { refractory SZ }\end{array}$ & 190 & 20 & 0.618 & 11 & 195 & 0.130 & 2 & 4 & 0.009 \\
\hline \multicolumn{10}{|l|}{ Other } \\
\hline Patients with HS $(N)$ & 50 & 13 & $<0.001$ & NA & NA & NA & NA & NA & NA \\
\hline $\begin{array}{l}\text { Onset age of } \\
\text { psychosis (y) }\end{array}$ & NA & $27.2 \pm 13.1$ & NA & NA & NA & NA & NA & NA & NA \\
\hline \multicolumn{10}{|l|}{ Brain-age estimation (y) } \\
\hline Mean brain-PAD $\pm \mathrm{SD}$ & $5.3 \pm 7.7$ & $10.9 \pm 7.8$ & $<0.001$ & $10.6 \pm 5.6$ & $5.8 \pm 8.1$ & 0.386 & $9.3 \pm 6.6$ & $22.0 \pm 11.3$ & NA \\
\hline
\end{tabular}

brain-PAD brain-predicted age difference, TLE-Non-P temporal lobe epilepsy without psychosis, TLE-P temporal lobe epilepsy with psychosis, $S Z$ seizures, MRI(-) Epi MRI-negative epilepsies, HS hippocampal sclerosis

Comparisons of the mean brain-PAD were analyzed by ANCOVA. All other $p$ values were generated by an unpaired $t$ test or Pearson's $\chi^{2}$ test Statistical comparison of the mean brain-PAD between JME and PME was not performed in consideration of the small sample size
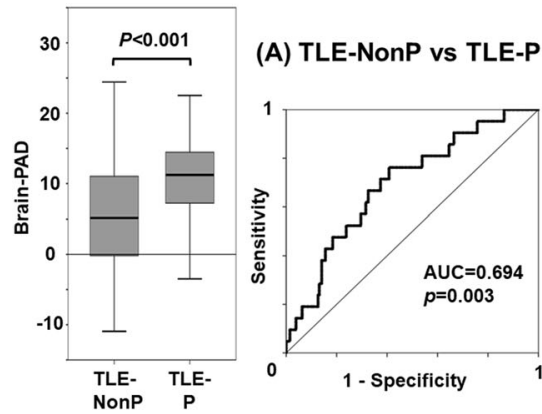

Fig. 2 The comparisons of the brain-PAD and discrimination of each clinical categorization. a TLE without psychosis (TLE-Non-P) vs.

A previous brain-age study of epilepsy showed a 4.5year increase in brain-PAD in 94 patients with refractory epilepsy [9]; this previous study consisted of various focus locations and partly included HS and FCD. This value would be consistent with our results in TLE with normal MRI or extra-temporal epilepsy groups (Table 2). Although they also reported no significant effect of HS on brain-PAD in their 12 patients with TLE and HS, our study, involving a higher number of samples $(N=63)$, suggested the significant effect of $\mathrm{HS}$ on the increased brain age.

The previous study also reported a significantly negative correlation of brain-PAD with onset age and no significant association with duration of disease [9]. We

\section{(B) PNES vs MRI(-) Epi}
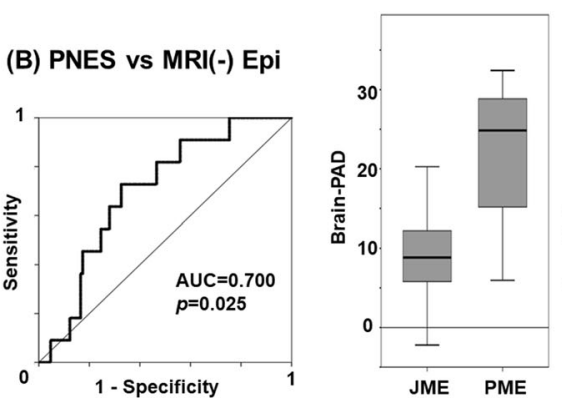

(C) JME vs PME

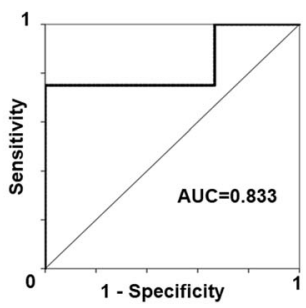

TLE with psychosis (TLE-P), b PNES vs. all MRI-negative epilepsies (MRI(-) Epi), and c JME vs. PME

also found the same correlation in the TLE-NL group (Supplementary Fig. 3b). This association between an increased brain-PAD and an earlier onset of epilepsy could suggest that the brain structural brain-age prediction may reflect the vulnerability to seizure generation or initial brain insult rather than disease progression. However, based on our results in each category, these correlations could depend on the type of epilepsy. In TLE-NL, conventional volumetry found no significant abnormality [24], despite the abnormal cortical thickness [25] and WM integrity [26]. Our voxel-based brain-age prediction may provide novel insights into structural neuroimaging in this group. 
In addition, we evaluated the effect of psychosis on TLE. The mean brain-PAD in TLE with psychosis was 10.9 years, which was significantly higher than that in TLE without psychosis (5.3 years). Considering the reported 5.5year increase in brain-PAD in schizophrenia [8], our result should be concordant with the comorbidity of psychosis and TLE. It might also suggest that the mechanism underlying the increased brain age in TLE would be different from that in psychosis. In fact, there is no solid agreement in brain volumetry in psychosis of epilepsy due to partly conflicting results of past studies [27] and there has recently been an attempt to detect network abnormalities beyond mere morphology [28]. Considering the importance of early detection and intervention for psychosis [29], more specific and individual-level biomarkers are desirable. The brain-age prediction was suggested as a potential biomarker of vulnerability to psychosis development [8] or accelerated neuromaturation [11]. Although a longitudinal survey is needed, we speculate that brain-age prediction could be a candidate biomarker of psychosis in epilepsy.

In addition, we focused on other types of epilepsy and PNES. Even the IGE patients with mostly controlled seizures showed higher values of the brain-PAD. Probably, the brain-PAD increase cannot simply be explained by refractory seizures and that, particularly in IGE, it might reflect the frontal lobe or thalamic dysfunction [30, 31]. Surprisingly, PNES patients with no electrophysiological seizures also showed a significantly increased brain-PAD, which was comparable with or even higher than that in MRInegative epilepsies. A recent neuroimaging review suggested functional and structural alterations, particularly related to emotion processing and cognitive-executive control, as a neurobiological mechanism in PNES [32]. This unstable cognitive-emotional-attention system could be associated with increased aging of the brain in PNES. The pathophysiology of PNES is still unclear, and our study may provide further information on it.

Furthermore, we also demonstrated a much greater increase in brain-PAD in PME compared with JME. This differentiation is still a clinical problem [16], and our results appear to agree with the differences between the two diseases, given the severe phenotype of PME [15]. However, the sample size in this comparison was small and patients with PME had a certain level of disease duration. Therefore, the current results cannot yield clear evidence on the utility of the brain age for differentiating myoclonic epilepsy in the early stages, although we believe that we have preliminarily shown its potential usefulness.

Moreover, we should discuss the underlying mechanisms of the altered brain age in epilepsy. Given that epilepsy is characterized by abnormal excessive neuronal activity (i.e., seizures) [1], recurrent electric damage may accelerate the brain aging in epilepsy. This scenario could be more applicable to our SGE/PME group, which represents the most severe category with extremely refractory, often daily, seizures, and intellectual deterioration. However, even the drug-responsive group (i.e., IGE) showed a certain level of increase in brain age. One potential explanation would be underlying predispositions in epilepsy, which might have affected patients' aging process throughout life including neurodevelopment. Another possible factor is inter-ictal epileptic discharges, which can be seen even in seizure-free cases, given the recent evidence of association with cognitive impairment [33]. In addition, recent evidence is revealing the progressive GM loss or abnormal tau deposition in epilepsy [34, 35]. Thus, our observation of increased brain aging is also consistent with a potentially neurodegenerative pathophysiology in epilepsy.

For clinical settings, brain age seems generally sensitive to the presence of epilepsy, but the within-group variability of brain-PAD is high. In addition, multiple nonepileptic conditions show an increased brain age [36]. Considering that the usefulness of brain age has been reported mostly for psychiatric disorders and cognitive impairment, brain age may have clinical potential for neurocognitive changes or psychiatric comorbidities in epilepsy. Furthermore, it is known that increased brain age predicts mortality risk [37]. Given that epilepsy is associated with an increased risk of sudden death [38], possibly related to specific brain structures essential for cardiorespiratory recovery [39], future application of brain age might be beneficial to identify patients at high risk of sudden death.

This study has several limitations. First, each group of patients had differing age/sex distributions, sample sizes, and diagnosis criteria, although we partly corrected for these differences using statistical methods. We should carefully interpret the current results, particularly those obtained in groups with a small sample size. Another limitation is the lack of psychiatric or psychological information other than psychosis, such as depression or intellectual disability. Given the high prevalence of intellectual disability in PNES [12], further investigations with neurocognitive data would be desirable, especially for PNES. Additionally, the cross-sectional design cannot answer questions about causal relationships or predictability in early stages. In particular, the usefulness for the differentiation of myoclonic epilepsy in the early stages is still unclear, considering the stage of our PME cohort. To address these limitations, further longitudinal validations with more detailed clinical information are needed.

In conclusion, we found increased brain-PAD in most types of epilepsy, with SGE/PME and TLE with HS showing particularly high values. In addition, TLE with psychosis presents a significant increase in brain-PAD compared with nonpsychotic TLE. Thus, brain-age prediction can provide a novel insight into or clinical usefulness for the diverse symptoms of epilepsy. 
Acknowledgements This study was supported by postdoctoral fellowship from Uehara Memorial Foundation to DS.

\section{Compliance with ethical standards}

Conflict of interest The authors declare that they have no conflict of interest.

Publisher's note: Springer Nature remains neutral with regard to jurisdictional claims in published maps and institutional affiliations.

Open Access This article is licensed under a Creative Commons Attribution 4.0 International License, which permits use, sharing, adaptation, distribution and reproduction in any medium or format, as long as you give appropriate credit to the original author(s) and the source, provide a link to the Creative Commons license, and indicate if changes were made. The images or other third party material in this article are included in the article's Creative Commons license, unless indicated otherwise in a credit line to the material. If material is not included in the article's Creative Commons license and your intended use is not permitted by statutory regulation or exceeds the permitted use, you will need to obtain permission directly from the copyright holder. To view a copy of this license, visit http://creativecommons. org/licenses/by/4.0/.

\section{References}

1. Fisher RS, van Emde Boas W, Blume W, Elger C, Genton P, Lee $\mathrm{P}$, et al. Epileptic seizures and epilepsy: definitions proposed by the International League Against Epilepsy (ILAE) and the International Bureau for Epilepsy (IBE). Epilepsia. 2005;46:470-2.

2. Scheffer IE, Berkovic S, Capovilla G, Connolly MB, French J, Guilhoto L, et al. ILAE classification of the epilepsies: position paper of the ILAE Commission for Classification and Terminology. Epilepsia. 2017;58:512-21.

3. Keezer MR, Sisodiya SM, Sander JW. Comorbidities of epilepsy: current concepts and future perspectives. Lancet Neurol. 2016;15: 106-15.

4. Berg AT, Altalib HH, Devinsky O. Psychiatric and behavioral comorbidities in epilepsy: a critical reappraisal. Epilepsia. 2017; 58:1123-30.

5. Arbabshirani MR, Plis S, Sui J, Calhoun VD. Single subject prediction of brain disorders in neuroimaging: promises and pitfalls. Neurilmage. 2017;145:137-65.

6. Bernhardt BC, Hong SJ, Bernasconi A, Bernasconi N. Magnetic resonance imaging pattern learning in temporal lobe epilepsy: classification and prognostics. Ann Neurol. 2015;77:436-46.

7. Gaser C, Franke K, Kloppel S, Koutsouleris N, Sauer H, Alzheimer's Disease Neuroimaging I. BrainAGE in mild cognitive impaired patients: predicting the conversion to Alzheimer's disease. PLoS ONE. 2013;8:e67346.

8. Koutsouleris N, Davatzikos C, Borgwardt S, Gaser C, Bottlender $\mathrm{R}$, Frodl $\mathrm{T}$, et al. Accelerated brain aging in schizophrenia and beyond: a neuroanatomical marker of psychiatric disorders. Schizophr Bull. 2014;40:1140-53.

9. Pardoe HR, Cole JH, Blackmon K, Thesen T, Kuzniecky R. Human Epilepsy Project I. Structural brain changes in medically refractory focal epilepsy resemble premature brain aging. Epilepsy Res. 2017;133:28-32.

10. Gaitatzis A, Trimble MR, Sander JW. The psychiatric comorbidity of epilepsy. Acta Neurol Scand. 2004;110:207-20.

11. Schnack HG, van Haren NE, Nieuwenhuis M, Hulshoff Pol HE, Cahn W, Kahn RS. Accelerated brain aging in schizophrenia: a longitudinal pattern recognition study. Am J Psychiary. 2016;173:607-16.

12. Kanemoto K, LaFrance WC Jr., Duncan R, Gigineishvili D, Park SP, Tadokoro Y, et al. PNES around the world: where we are now and how we can close the diagnosis and treatment gaps-an ILAE PNES Task Force report. Epilepsia Open. 2017;2:307-16.

13. Hingray C, El-Hage W, Duncan R, Gigineishvili D, Kanemoto K, LaFrance WC Jr., et al. Access to diagnostic and therapeutic facilities for psychogenic nonepileptic seizures: an international survey by the ILAE PNES Task Force. Epilepsia. 2018;59:203-14.

14. Brown RJ, Reuber M. Psychological and psychiatric aspects of psychogenic non-epileptic seizures (PNES): a systematic review. Clin Psychol Rev. 2016;45:157-82.

15. Berkovic SF, Andermann F, Carpenter S, Wolfe LS. Progressive myoclonus epilepsies: specific causes and diagnosis. N Engl J Med. 1986;315:296-305.

16. Badawy RA, Macdonell RA, Jackson GD, Berkovic SF. Can changes in cortical excitability distinguish progressive from juvenile myoclonic epilepsy? Epilepsia. 2010;51:2084-8.

17. American Psychiatric Association. DSM-IV Diagnostic and Statistical Manual of Mental Disorders. 4th ed. Washington, DC: American Psychiatric Press; 1994.

18. Ashburner J. A fast diffeomorphic image registration algorithm. Neuroimage. 2007;38:95-113.

19. Cole JH, Leech R, Sharp DJ, Alzheimer's Disease Neuroimaging I. Prediction of brain age suggests accelerated atrophy after traumatic brain injury. Ann Neurol. 2015;77:571-81.

20. Cole JH, Underwood J, Caan MW, De Francesco D, van Zoest RA, Leech R, et al. Increased brain-predicted aging in treated HIV disease. Neurology. 2017;88:1349-57.

21. Franke K, Ziegler G, Kloppel S, Gaser C, Alzheimer's Disease Neuroimaging I. Estimating the age of healthy subjects from T1weighted MRI scans using kernel methods: exploring the influence of various parameters. Neuroimage. 2010;50:883-92.

22. Lancaster J, Lorenz R, Leech R, Cole JH. Bayesian optimization for neuroimaging pre-processing in brain age classification and prediction. Front Aging Neurosci. 2018;10:28.

23. Faul F, Erdfelder E, Lang AG, Buchner A. G*Power 3: a flexible statistical power analysis program for the social, behavioral, and biomedical sciences. Behav Res Methods. 2007;39:175-91.

24. Mueller SG, Laxer KD, Cashdollar N, Buckley S, Paul C, Weiner MW. Voxel-based optimized morphometry (VBM) of gray and white matter in temporal lobe epilepsy (TLE) with and without mesial temporal sclerosis. Epilepsia. 2006;47:900-7.

25. Mueller SG, Laxer KD, Barakos J, Cheong I, Garcia P, Weiner MW. Widespread neocortical abnormalities in temporal lobe epilepsy with and without mesial sclerosis. Neuroimage. 2009;46:353-9.

26. Concha L, Beaulieu C, Collins DL, Gross DW. White-matter diffusion abnormalities in temporal-lobe epilepsy with and without mesial temporal sclerosis. J Neurol Neurosurg Psychiatry. 2009;80:312-9.

27. Allebone J, Kanaan R, Wilson SJ. Systematic review of structural and functional brain alterations in psychosis of epilepsy. J Neurol Neurosurg Psychiatry. 2018;89:611-7.

28. Sone D, Matsuda H, Ota M, Maikusa N, Kimura Y, Sumida K, et al. Graph theoretical analysis of structural neuroimaging in temporal lobe epilepsy with and without psychosis. PLoS One. 2016;11:e0158728.

29. Marshall M, Rathbone J. Early intervention for psychosis. Cochrane Database Syst Rev. 2011:CD004718.

30. Wandschneider B, Thompson PJ, Vollmar C, Koepp MJ. Frontal lobe function and structure in juvenile myoclonic epilepsy: a comprehensive review of neuropsychological and imaging data. Epilepsia. 2012;53:2091-8.

31. Sone D, Watanabe M, Ota M, Kimura Y, Sugiyama A, Maekawa T, et al. Thalamic hypoperfusion and disrupted cerebral 
blood flow networks in idiopathic generalized epilepsy: arterial spin labeling and graph theoretical analysis. Epilepsy Res. 2017;129:95-100.

32. Asadi-Pooya AA. Neurobiological origin of psychogenic nonepileptic seizures: a review of imaging studies. Epilepsy Behav. 2015;52:256-9.

33. Glennon JM, Weiss-Croft L, Harrison S, Cross JH, Boyd SG, Baldeweg T. Interictal epileptiform discharges have an independent association with cognitive impairment in children with lesional epilepsy. Epilepsia. 2016;57:1436-42.

34. Tai XY, Koepp M, Duncan JS, Fox N, Thompson P, Baxendale S, et al. Hyperphosphorylated tau in patients with refractory epilepsy correlates with cognitive decline: a study of temporal lobe resections. Brain. 2016;139:2441-55.
35. Caciagli L, Bernasconi A, Wiebe S, Koepp MJ, Bernasconi N, Bernhardt BC. A meta-analysis on progressive atrophy in intractable temporal lobe epilepsy: Time is brain? Neurology. 2017;89:506-16.

36. Cole JH, Marioni RE, Harris SE, Deary IJ. Brain age and other bodily 'ages': implications for neuropsychiatry. Mol Psychiatry. 2019;24:266-81.

37. Cole JH, Ritchie SJ, Bastin ME, Valdes Hernandez MC, Munoz Maniega S, Royle N. et al. Brain age predicts mortality. Mol Psychiatry. 2018;23:1385-92.

38. Tomson T. Mortality in epilepsy. J Neurol. 2000;247:15-21.

39. Allen LA, Vos SB, Kumar R, Ogren JA, Harper RK, Winston GP. et al. Cerebellar, limbic, and midbrain volume alterations in sudden unexpected death in epilepsy. Epilepsia 2019;60:718-29. 